\title{
A multi-physics peridynamics-DEM-IB-CLBM framework for the prediction of erosive impact of solid particles in viscous fluids
}

\author{
Ya Zhang ${ }^{\mathrm{a}, \mathrm{b}}$, Guang Pan ${ }^{\mathrm{a}, \mathrm{b}, *}$, Yonghao Zhang ${ }^{\mathrm{c}}$, Sina Haeri ${ }^{\mathrm{c}, *}$, \\ ${ }^{a}$ School of Marine Science and Technology, Northwestern Polytechnical University, \\ Xian, 710072, China \\ ${ }^{b}$ Key Laboratory of Unmanned Underwater Vehicle, Northwestern Polytechnical University, \\ Xian, 710072, China \\ ${ }^{c}$ James Weir Fluids Laboratory, Department of Mechanical and Aerospace Engineering, \\ University of Strathclyde, Glasgow, UK, G1 $1 X J$
}

\begin{abstract}
In this paper, a new fully-resolved framework capable of capturing the fundamental physics of particle-fluid interactions, the collision of particles with solid surfaces, and the resulting damage is proposed. A coupled DEM-IB-CLBM, consisting of a discrete element method (DEM), an immersed boundary (IB) method, and a cascaded lattice Boltzmann method (CLBM), is used to fully resolve the interaction of the particles with the surrounding viscous fluid. The peridynamics theory is then implemented and used to predict the impact damage to the target material. This framework is validated by comparing the trajectory of a particle-wall collision event in a viscous fluid with the previous results in the literature. Furthermore, the variation of the restitution coefficient with the impact velocity is in a good agreement with the available experimental results. The influence of multiple impacts and the resulting surface damage on the fluid dynamics of the system is investigated. It is demonstrated that the method correctly predicts the expected effects of multiple collisions and impact angle variations on the surface damage.
\end{abstract}

Keywords: Erosion, Particle impact, Immersed boundary method, Discrete element method, Fully resolved simulations, Cascaded lattice Boltzmann method

\section{Introduction}

The presence of solid particles (such as sands) in many flow instruments and pipelines, leads to major erosion problems such as degradation of pipelines and

\footnotetext{
* Corresponding authors

Email addresses: panguang@nwpu.edu.cn (Guang Pan ), sina.haeri@strath.ac.uk (Sina Haeri )
}

Preprint submitted to Computer Methods in Applied Mechanics and EngineeringMay 22, 2019 
production equipment including pumps and valves. The impinging particles 5 will cause excessive damage to the surface layer, which will also reduce the effectiveness of corrosion inhibitors resulting in material degradation. Meanwhile, corrosion will in turn accelerate the erosion rate resulting in severe and rapid loss of surface metal (washout). The material defect may shorten the lifetime of pipelines and increase production risk and lack of predictive design tools makes to prevention of catastrophic failure difficult.

The erosion by solid particle impact is a complex phenomenon and involves the interplay of several factors including flow pattern and geometry, fluid properties, particle size/shape distribution, and particle/surface material characteristics [1 3. The standard finite-volume computational fluid dynamics (CFD)

15 methods coupled with un-resolved particle tracking (Eulerian-Lagrangian) and erosion models have been employed [4/7) to predict erosion rates in different geometries. In these studies, CFD techniques are used to solve the flow field and track particles. However, in this approach, the fluid forces on the particles are estimated based on semi-empirical models. Moreover, the erosion rate is 20 estimated purely based on empirical models.

Comprehensive reviews of particle erosion modelling techniques and the dynamics of the erosion process caused by the continuous particle impingement have been provided in the literature [3, 8, 10]. The general consensus is that the available empirical models should be used with utmost care since they fail

25 to include the effects of important parameters such as particle shape and rotational velocity. Furthermore, statistical inaccuracies exist in developing these models due to the scarcity of experimental data. Therefore, development of a framework to simulate particle erosion based on the first principles is urgently required.

30 Several challenges have hindered the development of a high-fidelity framework, which includes calculation of material deformation and crack propagation, modelling the deforming material surface immersed in the fluid, and dynamical coupling between the material and flow field. In order to consider these effects simultaneously in a simulation, naturally, a multi-physics framework is 35 required: a coupled numerical method that can solve the flow field, particle impacts, material damage and interactions among them. In this paper, the fluid flow, the immersed boundaries of rigid particles and the deformable material surfaces are modelled using a recently developed hybrid method which couples discrete element, immersed boundary, and cascaded lattice Boltzmann methods

40 (DEM-IB-CLBM) 11. It has been fully validated and offers a reliable framework to simulate the particulate flow problems. However, an accurate technique to predict the material damage is still required.

The peridynamics (PD) theory is an innovative approach for modelling material damage and fractures, where the partial differential equations of the classical 45 continuum mechanics fail. In this theory, the partial differential equations of the continuum is recast as an integral equation. The theory was first purposed by Silling [12] and applied to impact damage predictions [13. The numerical method introduces the concept of non-local interactions between material points [13, 14] to express the internal interactions within the solid, which assumes that 
${ }_{50}$ a point within the material is subjected to forces from all points in its neighbourhood. PD has been utilized successfully for material deformation and damage prediction problems. Silling and Askari [13] predicted impact damages including simulations of a Charpy $\mathrm{V}$-notch test, accumulated damage in concrete due to multiple impacts, and crack fragmentation of a glass plate. Oterkus et al.

55 [15] presented an application for damage assessment and residual strength of a reinforced panel under compression after impact due to a rigid penetrator. Tupek [16] extended PD simulations to material exposed to extreme loads, such as Taylor and ballistic impacts. Madenci and Oterkus 17 presented an ordinary state-based peridynamics constitutive relations for plastic deformation and validated their predictions by considering several benchmark problems.

PD is a flexible theory and can be implemented using a "particle-based" approach. Therefore, it can be coupled naturally with our DEM-IB-CLBM approach, to yield a new, fully-resolved particle-fluid-material interaction framework: PD-DEM-IB-CLBM. It can capture all the fundamental physical phenomena involved in the particle erosion process. The framework only relies on the first principles which rids it from ad hoc models to provide an unprecedented insight into the impact dynamics and the resulting material damage. In this first paper, our emphasis is on the development of a new numerical framework and the algorithm efficiency is not the primary concern. In addition, to man-

70 age the computational costs we completely remove the chipped surface material from the simulations. However, this is not a limitation of the current framework and in the future, a threshold can be introduced to only remove chipped material of certain sizes. The present peridynamics implementation can only handle the brittle materials. However, with the rapid development of PD more

75 sophisticated and realistic models for ductile material can be included in this framework.

The paper is organised as follows. In Section 2, the peridynamics theory, and the DEM-IB-CLBM approach are succinctly introduced and the coupled algorithm is provided in detail. In Section 3, the new method is validated by

so considering the classical single particle-wall collision problem. The rebound behaviour and the restitution coefficient for the impact of a rigid particle with different sizes and velocities are determined and compared with the experimental results. In Section 4, the coupled method is employed to study the erosion caused by single and multiple particle-wall collisions in a viscous fluid and the concluding remarks are provided in Section 5 .

\section{Methodology}

\subsection{Peridynamics theory}

Compared to the classical formulation of solid mechanics, which is based on partial differential equations, the peridynamics theory uses an integral equation to describe the relative displacements and forces between neighbouring material points (i.e. the particles that represents the solid material) and hence it naturally captures the discontinuities, such as cracks and material damages [13. 
This advantage combined with the fact that it can be implemented using a "particle-based" technique renders the theory as a promising numerical tool to study the particle erosion problem. Here we discuss the theory briefly and the reader is referred to Silling and Askari [13, Madenci and Oterkus [18 for details. The PD theory formulates the motion of material points as

$$
\rho_{m} \ddot{\mathbf{d}}(\mathbf{x}, t)=\int_{H} \mathbf{f}(\eta, \xi) d V_{\mathbf{x}^{\prime}}+\mathbf{F}_{b}(\mathbf{x}, t),
$$

where $\rho_{m}$ is the density of material points and $\mathbf{d}$ is their displacement vector of a point located at $\mathbf{x}$ at time $t$. A particle based approach is used to implement 90 the PD and hence the material is discretised using a set of particles. Therefore, material points are interchangeably referred to as PD particles. The time derivative of displacement of each PD particle $\ddot{\mathbf{d}}$ is related to the integral of an internal force field $\mathbf{f}(\eta, \xi)$ and an external body force $\mathbf{F}_{b}$. The neighbourhood of the material point at $\mathbf{x}$ is called a horizon and is indicated by $H$, which is 95 assumed to be a circular (spherical in $3 \mathrm{D}$ ) region of radius $\delta$ in this paper. The force exerted on the material point $\mathbf{x}$ by all the points $\mathbf{x}^{\prime}$ within $H$ is the integral of a force density $\mathbf{f}(\eta, \xi)$ over the volume $V_{\mathbf{x}^{\prime}}$, where $\xi=\mathbf{x}^{\prime}-\mathbf{x}$ and $\eta=\mathbf{d}^{\prime}-\mathbf{d}$ are the relative position and displacement vectors respectively.

A prototype micro-elastic brittle (PMB) material model [13] is used to represent inter-particle bonds in our particle-based implementation of the theory. The force density takes the form

$$
\mathbf{f}(\eta, \xi)=c s \phi \mathbf{n},
$$

where for two-dimensional structures the bond constant is given by

$$
c=9 E / \pi h \delta^{3},
$$

and is related to the Young's modulus $E$ and structure thickness $h$. In Eq. (2), $\phi$ is a history dependent function that is related to the material damage which will be explained later in this section and the unit vector $\mathbf{n}$ points from $\mathbf{x}+\mathbf{d}$ to $\mathbf{x}^{\prime}+\mathbf{d}^{\prime}$.

The scalar bond stretch $s$ is defined as

$$
s=\frac{|\eta+\xi|-|\xi|}{|\xi|} .
$$

If the bond stretch $s$ exceeds its critical value $s_{c}$, the bond breaks irreversibly. This treatment allows material damage to be simulated at the bond level. The critical stretch $s_{c}$ is obtained from

$$
s_{c}=\sqrt{\frac{4 G_{c}}{h c \delta^{4}}}
$$

where $G_{c}$ is the fracture energy of the material. 
The accumulation of broken bonds leads to material separation and the formation of cracks. Local damage of a material point $d_{i}$ is defined as the ratio of the number of broken bonds to the total number of bonds,

$$
d_{i}(\mathbf{x}, t)=1-\frac{\int_{H} \phi(\eta, \xi) d V_{\mathbf{x}^{\prime}}}{\int_{H} d V_{\mathbf{x}^{\prime}}} .
$$

The damage $d_{i} \in[0,1]$, where $d_{i}=0$ means an undamaged material point and $d_{i}=1$ denotes a completely damaged point which has lost all its bonds.

The material points in contact with the fluid should be identified in order to impose the no-slip boundary conditions on them to calculate the fluid flow. Therefore, the damage $d_{i}$ is calculated throughout the material domain, and its value is used as an indicator of material points in contact with the fluid. The material points in the vicinity of a fracture will have a large local damage $d_{i}$ due to the broken bonds. To efficiently identify the surface points we define a threshold value $d_{s}=0.35$. If any material point has $d_{i} \geq d_{s}$, then the material point is marked as a possible surface point.

When $d_{i}=1.0$ the material point has lost all of its bonds. Such a point is essentially a freely moving particle (chipped material) in the fluid flow. It is possible to use adaptive grid refinement around these newly generated particles and to fully resolve them using the immersed boundary method. However, since the size of these particles is in order of $\mathcal{O}(\Delta)$, resolving all of them will substantially increase the computational time. Therefore, in the current algorithm when the local damage $d_{i}$ is larger than a critical value $d_{c}=0.8$, the point is permanently removed from the simulation by cutting all its connections to other material points and its local damage is set to $d_{i}=1.0$. The total damage of material $D_{t o t}$ is calculated by summing the local damages $d_{i}$ of all the material points including the points removed from the simulation,

$$
D_{t o t}(t)=\sum d_{i}(\mathbf{x}, t) .
$$

In a bond-based implementation, the relative positions between material points are controlled by the bond forces and when the two points come close to each other, they will repel to prevent inter-penetration. However, most of the PD bonds near fractures are broken, and inter-penetration of points may occur under a compression. This problem can be circumvented by adding short-range forces between neighbouring material points [13. However this method requires an ad hoc short-range force intensity and generates numerical artefacts. To address these issues, in this work the history dependant function $\phi$ is modified instead as

$$
\phi= \begin{cases}1, & \text { for live bonds and broken bonds with } s \leq 0 \\ 0, & \text { otherwise }\end{cases}
$$

which is motivated by the ideas presented by Tupek [16. This history-dependent function activates the force contribution of a damaged bond when it is under 
compression and breaks only if it is under tension. It allows the algorithm to effectively deal with the inter-penetration issue of the nearly separated material points.

\subsection{The DEM-IB-CLBM approach}

In the proposed multi-physics framework, rigid impactors move in a fluid and hit deformable materials with an impact velocity. This leads to material damage and consequently an algorithm is required to deal with the deforming boundaries of the fluid domain. Here our recent DEM-IB-CLBM approach 11 is adopted to accurately simulate the fluid flow and its interactions with the solid objects. The DEM-IB-CLBM method is succinctly discussed in this section for completeness and the reader is referred to [11] for further details. The cascaded lattice Boltzmann method provides the flow field while the immersed boundary method efficiently enforces the no-slip condition on the moving boundaries of the rigid impactors and deformable material surfaces using a moving Lagrangian grid. The discrete element method (DEM) deals with the possible collisions between impactors. The evolution of the DEM-IB-CLBM approach from time $t$ to $t+\delta t$ is explained here.

In the CLBM, the fluid is described by a set of imaginary particles, which stream along a uniform lattice grid at the fixed reference frame and collide with each other on a moving frame. The fluid is modelled with a set of distribution functions which evolve according to the following equation:

$$
\mathbf{f}(\mathbf{x}+\mathbf{e} \delta t, t+\delta t)=\mathbf{f}(\mathbf{x}, t)+\mathbf{\Omega}(\mathbf{x}, t)+\mathbf{S} \delta t .
$$

In Eq. (9), $\mathbf{f}$ is the vector of the particle distribution functions representing the probability of finding a particle at the position $\mathbf{x}$ with the discrete velocity $\mathbf{e}$ at the time $t$. Furthermore, $\boldsymbol{\Omega}, \mathbf{S}$ and $\delta t$ are respectively the discrete collision operator, the discrete force term and the time step. The CLBM algorithm employs a split-forcing model [19] which takes the body forcing term into account through a collision and a velocity modification step. The fluid density $\rho$ and the unmodified velocity $\mathbf{u}^{*}$ are obtained by taking the zero ${ }^{\text {th }}$ and first moments of the distribution function as follows,

$$
\begin{aligned}
\text { CLBM macros: } \rho(\mathbf{x}, t) & =\sum_{\alpha=0}^{8} f_{\alpha}(\mathbf{x}, t), \\
\mathbf{u}^{*}(\mathbf{x}, t) & =\frac{1}{\rho(\mathbf{x}, t)} \sum_{\alpha=0}^{8} \mathbf{e}_{\alpha} f_{\alpha}(\mathbf{x}, t) .
\end{aligned}
$$

In the DEM-IB-CLBM approach, the fluid domain is discretised using a fixed Eulerian mesh and the surface of solids is represented by a moving Lagrangian grid. In this paper, the variables in the Lagrangian frame are represented by a subscript $l$ and the variables in the Eulerian frame have no subscript. In the IBM simulation, a no-slip boundary is enforced by calculating a forcing term $\mathbf{F}_{i b, l}$ on the Lagrangian points $\mathbf{x}_{l}$ by using the unmodified velocity field $\mathbf{u}^{*}$ and 
then distributing $\mathbf{F}_{i b, l}$ onto the surrounding fluid nodes $\mathbf{x}$ using a discrete delta function with a compact support [20, 21. To accurately implement the noslip boundary conditions, a cumulative multi-direct-forcing (MDF) scheme is used [11. The iteration is initialized with $\mathbf{u}^{0}(\mathbf{x}, t)=\mathbf{u}^{*}(\mathbf{x}, t), \mathbf{F}_{i b, l}^{0}\left(\mathbf{x}_{l}, t\right)=0$, $\mathbf{F}_{i b}^{0}(\mathbf{x}, t)=0$ and the counter is set to $k=0$. Then the following equations are solved successively during each iteration of the cumulative MDF scheme:

$$
\begin{aligned}
\text { IBM Interpolation: } \mathbf{u}^{k}\left(\mathbf{x}_{l}, t\right) & =\sum_{\mathbf{x}} \mathbf{u}^{k}(\mathbf{x}, t) \Phi\left(\mathbf{x}-\mathbf{x}_{l}, t\right) \delta x^{2}, \\
\Delta \mathbf{u}_{l}^{k}\left(\mathbf{x}_{l}, t\right) & =\mathbf{u}_{l}\left(\mathbf{x}_{l}, t\right)-\mathbf{u}^{k}\left(\mathbf{x}_{l}, t\right), \\
\mathbf{F}_{i b, l}^{k}\left(\mathbf{x}_{l}, t\right) & =\mathbf{F}_{i b, l}^{k-1}\left(\mathbf{x}_{l}, t\right)+\frac{2 \rho \Delta \mathbf{u}_{l}\left(\mathbf{x}_{l}, t\right)}{\delta t} ; \\
\text { IBM Distribution: } \Delta \mathbf{u}^{k}(\mathbf{x}, t) & =\sum_{\mathbf{x}_{l}} \Delta \mathbf{u}_{l}^{k}\left(\mathbf{x}_{l}, t\right) \Phi\left(\mathbf{x}-\mathbf{x}_{l}\right) d s_{l}, \\
\mathbf{u}^{k+1}(\mathbf{x}, t) & =\mathbf{u}^{k}(\mathbf{x}, t)+\Delta \mathbf{u}^{k}(\mathbf{x}, t), \\
\mathbf{F}_{i b}^{k}(\mathbf{x}, t) & =\mathbf{F}_{i b}^{k-1}(\mathbf{x}, t)+\frac{2 \rho \Delta \mathbf{u}(\mathbf{x}, t)}{\delta t}
\end{aligned}
$$

where $d s_{l}$ is the spacing between the Lagrangian points and the iteration stops after $k=5$ which is found to provide a trade-off between computational accuracy and cost [11.

The converged body force $\mathbf{F}_{i b}(\mathbf{x}, t)$, is then used in the collision and streaming operations to update the fluid field as follows:

$$
\begin{aligned}
& \text { CLBM colliding: } \mathbf{f}^{\prime}(\mathbf{x}, t)=\mathbf{f}(\mathbf{x}, t)+\mathbf{\Omega}(\mathbf{x}, t)+\mathbf{S} \delta t, \\
& \text { CLBM steaming: } \mathbf{f}(\mathbf{x}+\mathbf{e} \delta t, t+\delta t)=\mathbf{f}^{\prime}(\mathbf{x}, t),
\end{aligned}
$$

where $\mathbf{f}^{\prime}$ is the post-collision distribution function.

The centre of mass $\mathbf{X}$ and the angle of rotation $\boldsymbol{\Theta}$ of the particle are updated according to the rigid body equations of motion which are discretised as follows [11, 22]:

$$
\begin{aligned}
\text { DEM update: } \mathbf{X}(t+\delta t) & =2 \mathbf{X}(t)-\mathbf{X}(t-\delta t)+\frac{\mathbf{F}^{p}(t)}{m_{p}} \delta t^{2}, \\
\mathbf{\Theta}(t+\delta t) & =2 \boldsymbol{\Theta}(t)-\mathbf{\Theta}(t-\delta t)+\frac{\mathbf{T}^{p}(t)}{I_{p}} \delta t^{2}, \\
\mathbf{U}(t+\delta t) & =\frac{\mathbf{X}(t+\delta t)-\mathbf{X}(t-\delta t)}{2 \delta t}, \\
\boldsymbol{\Omega}(t+\delta t) & =\frac{\boldsymbol{\Theta}(t+\delta t)-\boldsymbol{\Theta}(t-\delta t)}{2 \delta t},
\end{aligned}
$$

where $m_{p}$ and $I_{p}$ are the mass and the moment of inertia of the rigid impactor respectively. Furthermore, $\mathbf{U}(t)$ and $\boldsymbol{\Omega}(t)$ are the displacement and angular velocities. The velocity and location of the Lagrangian points $\mathbf{u}_{l}\left(\mathbf{x}_{l}, t\right)$ and $\mathbf{x}_{l}$ located on the surface of rigid particles are updated after the location and 


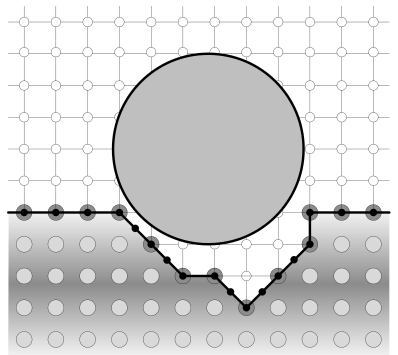

Figure 1: An illustration of the coupled PD-DEM-IB-CLBM scheme. The LBM lattices: O; the IBM points: •; the PD material points: $\bigcirc$; the material surface points: $\bigcirc$.

velocity of particle's centre are determined. In Eqs. $20-(23), \mathbf{F}^{p}$ and $\mathbf{T}^{p}$ are the force and torque acting on the particle's centre of mass. The force $\mathbf{F}^{p}$ and torque $\mathbf{T}^{p}$ are calculated via

$$
\begin{aligned}
& \mathbf{F}^{p}=\mathbf{F}_{h}^{p}+\mathbf{F}_{c}+\mathbf{G}, \\
& \mathbf{T}^{p}=\mathbf{T}_{h}^{p}+\mathbf{T}_{c},
\end{aligned}
$$

where $\mathbf{F}_{h}^{p}$ and $\mathbf{F}_{c}$ are hydrodynamics and collision forces, respectively; $\mathbf{T}_{h}^{p}$ and $\mathbf{T}_{c}$ are the corresponding torques; and $\mathbf{G}$ is the gravity.

In the DEM-IB-CLBM approach, the hydrodynamic force $\mathbf{F}_{h}^{p}$ acting on a rigid particle is directly calculated by adding the force contributions $\mathbf{F}_{i b, l}$ from all the Lagrangian points that represent the particle's surface:

$$
\mathbf{F}_{h}^{p}(t)=\sum-\mathbf{F}_{i b, l}\left(\mathbf{x}_{l}, t\right) d s_{l}+\mathbf{F}_{i n},
$$

where $\mathbf{F}_{\text {in }}$ represents the effect of internal mass 23. The DEM approach is used to prevent interpenetration of solid particles and to accurately resolve the collisions between them. Here, a spring-dashpot contact model is used to obtain the collision force $\mathbf{F}_{c}^{\text {dem }}$. In addition, a time marching scheme is required in DEM to integrate the Newton's equations of motion. The step size condition $\delta t^{d e m}<\delta t_{c}^{d e m}$ should be satisfied during the time marching in order to properly resolve the collisions [24, 25].

\subsection{The coupled peridynamics and DEM-IB-CLBM approach}

A wide range of length and time scales are relevant in fracture dynamics of material. A large-scale fracture can be captured easily while hairline cracks can only be reproduced with a high-fidelity simulation. Here, the assumed size of the PD material points controls the resolution. In this work, we aim to demonstrate the feasibility of PD-DEM-IB-CLBM and for convenience, the size of the PD material point $\Delta$ is the same as the lattice spacing $\delta x$ used for CLBM. Moreover, the locations of material points $\mathbf{x}$ are initialised to coincide with the lattice points as shown in Figure 1. 
The system in Eq. 1 has been discretised in both the space and the time as follows [14:

$$
\rho_{m} \frac{\mathbf{d}(\mathbf{x})^{n+1}-2 \mathbf{d}(\mathbf{x})^{n}+\mathbf{d}(\mathbf{x})^{n-1}}{\delta t^{p d^{2}}}=\sum_{\mathbf{x}^{\prime}} \mathbf{f}^{n}(\eta, \xi) V_{\mathbf{x}^{\prime}}+\mathbf{F}_{b}^{n}(\mathbf{x}), \text { for }\left\|\mathbf{x}-\mathbf{x}^{\prime}\right\| \leq \delta,
$$

where a constant time step $\delta t^{p d}$ is employed for transient dynamic simulations and $n$ is the current time step. The numerical stability condition of Eq. 27 leads to a critical time step $\delta t_{c}^{p d}$ which for a two-dimensional structure can be estimated by:

$$
\delta t_{c}^{p d}=0.8 \sqrt{\frac{2 \rho_{m}}{\pi \delta^{2} c}}
$$

where $\delta=3.015 \Delta[18$.

The interaction between different components of the coupled framework is presented in Fig. 2. Here, the IBM acts as an interface that connects the other three solvers together, i.e. the DEM, PD, and the CLBM modules. This is achieved by calculating three forces, the immersed boundary force $\mathbf{F}_{i b}$ for enforcing the no-slip condition at the surface of particles and solid walls, the hydrodynamic force $\mathbf{F}_{h}^{p}$ acting on rigid impactors, and a hydrodynamic force density acting on the material surface points $\mathbf{F}_{h}^{p d}$ that contributes to $\mathbf{F}_{b}$ on the RHS of Eq. 27. Furthermore, $\mathbf{F}_{h}^{p}$ is computed from $\mathbf{F}_{i b}$ using Eq. 26. The hydrodynamic force on the material surface points is equal and opposite to the force exerted on the fluid by the immersed boundary and $\mathbf{F}_{h}^{p d}$ is calculated by

$$
\mathbf{F}_{h}^{p d}=-\mathbf{F}_{i b} \frac{d s_{l} \delta x}{V_{\mathbf{x}}}
$$

The locations and velocities of rigid particles and material surface points, $\mathbf{X}^{p}$ and $\mathbf{V}^{p}, \mathbf{X}^{p d}$ and $\mathbf{V}^{p d}$, are used to calculate the location and velocities of the Lagrangian points for the IB module (i.e. $\mathbf{x}_{l}$ and $\mathbf{u}_{l}\left(\mathbf{x}_{l}\right)$ ). The no-slip boundary conditions on the surface of rigid particles and materials can now be enforced using the immersed boundary forces $\mathbf{F}_{i b}$ which is computed using the velocity and position of the Lagrangian points and the flow information computed by the CLBM module as discussed in Section 2.2.

The interaction between DEM and PD is demonstrated in Fig. 2 and represents the physical contact between rigid impactors and deformable brittle material whose motion is governed by the peridynamics equations. During a particle-surface impact, the material point $\mathbf{x}$ that falls inside an impactor will be relocated to the outside, and a corresponding collision force density $\mathbf{F}_{c}^{p d}(\mathbf{x})$ is computed which is then embedded in $\mathbf{F}_{b}$ [18. Moreover, the opposite force $-\mathbf{F}_{c}^{p d}(\mathbf{x}) V_{\mathbf{x}}$ will act on the impactor and is added to the particle's collision force $\mathbf{F}_{c}$ to predict its motion through (Eq. $(20)-(23)$ ). Therefore, the collision force in Eq. 24 is composed of two parts, the collision forces with other impactors $\mathbf{F}_{c}^{\text {dem }}$ and those from contact with the material points:

$$
\mathbf{F}_{c}=\mathbf{F}_{c}^{d e m}-\sum_{\mathbf{x}} \mathbf{F}_{c}^{p d}(\mathbf{x}) V_{\mathbf{x}}
$$




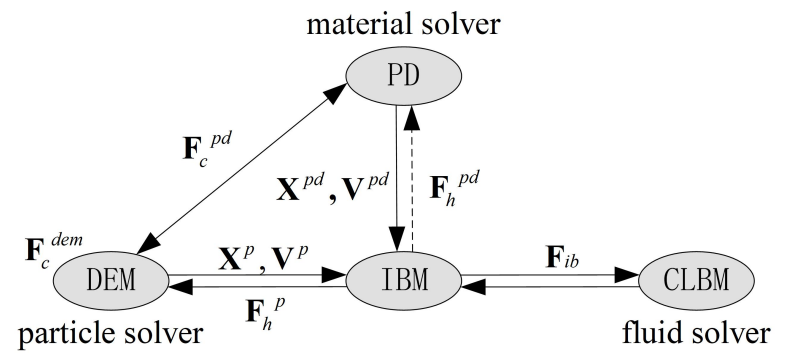

Figure 2: The interaction between different solvers in the coupled framework.

The evolution of the PD points in the coupled framework is then performed as follows,

$$
\begin{aligned}
& \text { PD update: } \\
& \begin{aligned}
\mathbf{F}_{p d}(\mathbf{x}, t)=\sum_{\mathbf{x}^{\prime}} \mathbf{f}(\eta, \xi, t) V_{\mathbf{x}^{\prime}}, \text { for }\left\|\mathbf{x}-\mathbf{x}^{\prime}\right\| \leq \delta, \\
\mathbf{F}_{b}(\mathbf{x}, t)=\mathbf{F}_{h}^{p d}+\mathbf{F}_{c}^{p d}, \\
\dot{\mathbf{d}}(\mathbf{x}, t+\delta t)=\dot{\mathbf{d}}(\mathbf{x}, t)+\frac{\delta t}{\rho_{m}}\left(\mathbf{F}_{p d}(\mathbf{x}, t)+\mathbf{F}_{b}(\mathbf{x}, t)\right), \\
\mathbf{d}(\mathbf{x}, t+\delta t)=\mathbf{d}(\mathbf{x}, t)+\delta t \dot{\mathbf{d}}(\mathbf{x}, t+\delta t) .
\end{aligned}
\end{aligned}
$$

\subsection{The PD-DEM-IB-CLBM algorithm}

Most brittle materials have a relatively high Young's module $E$ and a sud-

165 time step $\delta t_{c}^{p d}$ given by Eq. 3 and Eq. 28 enforces a very small step for capturing the material's response, otherwise numerical instabilities will occur during the time marching scheme presented in Eqs. (31)-(34). Moreover, the time step of the DEM-IB-CLBM module $\delta t$ should be small enough to capture the ever, the collision time scale is usually much smaller than the fluid flow time scale. Therefore, to seamlessly integrate the PD solver with the DEM-IB-CLBM module we require that $\delta t<\min \left(\delta t_{c}^{p d}, \delta t_{c}^{d e m}\right)$. The large step size disparity (i.e. $\left.\min \left(\delta t_{c}^{p d}, \delta t_{c}^{d e m}\right) \ll \delta t^{f l u i d}\right)$ will substantially increase the computational costs.

175 However, noting that such a small $\delta t$ is unnecessary for most of the fluid domain, a dual time marching scheme is employed to allow for the optimization of the framework for large-scale simulations which is explained as follows.

Since the PD and DEM solvers have frequent interactions, a time step $\delta t^{p d}<$ $\min \left(\delta t_{c}^{p d}, \delta t_{c}^{d e m}\right)$ is assigned to both solvers. Meanwhile, the IB-CLBM module uses a sufficiently small time step $\delta t$ to accurately capture the fluid flow. The relationship between these time steps is $\delta t=N \delta t^{p d}$, which means the flow field is updated by IB-CLBM after $N$ times of PD-DEM evolutions in the full framework. The step-by-step algorithm of the full framework can be summarized as follows: 
2. IBM Module: The IBM enforces the no-slip boundaries at the solid surface with Eqs. 12 17 then obtain the hydrodynamic forces $\mathbf{F}_{h}^{p}$ and the force density $\mathbf{F}_{h}^{p d}$ according to Eq. 26 and Eq. 29 , respectively;

3. CLBM Module: perform the colliding and streaming operations, Eq. 18 and Eq. 19.

4. Sub-evolutions of the PD and DEM;

(a) Find the values $\delta t^{p d}$, choose $N$ and set $i=0$;

(b) PD Module:

i. Compute the internal forces $\mathbf{F}_{p d}$ according to Eq. 31 .

ii. Detect rigid particle-wall collision and compute $\mathbf{F}_{c}^{p d}$;

iii. Update the displacement of material points $\mathbf{d}$ according to Eqs. 32 34

(c) DEM Module:

i. Detect particle-particle contact and compute $\mathbf{F}_{c}^{\text {dem }}$ using the spring-dashpot model;

ii. Calculate $\mathbf{F}_{c}$ according to Eq. [30, compute $\mathbf{F}^{p}$ and $\mathbf{T}^{p}$ in Eq. 24 and Eq. 25.

iii. Update the motion of rigid particles with Eq. 20 to Eq. 23

(d) Increase $i$ and repeat $4 \mathrm{~b}$ and $4 \mathrm{c}$ while $i<N$;

\section{Validation}

\subsection{Particle-wall collision in a viscous fluid}

215

The coupled framework is validated considering the particle-wall collision problem, as shown in Fig 3 . The standard gravity $\mathrm{g}$ is $981 \mathrm{~cm} / \mathrm{s}^{2}$, the density 
and the dynamic viscosity of fluid are $0.935 \mathrm{~g} / \mathrm{cm}^{3}$ and $0.1 \mathrm{~cm}^{2} / \mathrm{s}$, respectively. There are 50 lattice points across the rigid particle's diameter, $D=50 \Delta$. The rectangular computational domain is of size $(x=10 D, y=10 D)$, where the initial fluid domain is placed above the $x$ axis and a material plate with size $(10 D, 2 D)$ is below the $x$ axis. The particle centre is placed at $(5 D, 2 D)$. In an initial quiescent fluid domain, the zero-gradient boundary condition is applied in all the boundaries of the computational domain.

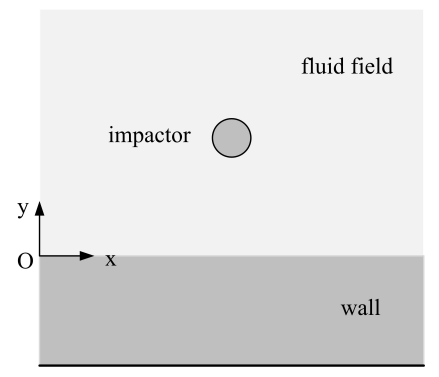

Figure 3: A schematic presentation of the particle-wall collision problem set up.

A dry restitution coefficient of $r_{c}=0.97$ is required to compare the particle rebound velocity to the results of Ardekani and Rangel [26. $r_{c}$ is computed as $r_{c}=V_{r} / V_{i m p}$, where $V_{r}$ is the rebound velocity of the particle and $V_{i m p}$ is the impact velocity. However, when solving for the material deformation with $\mathrm{PD}$, the restitution coefficient $r_{c}$ and the contact duration $T_{c}$ are not independent parameters but are the consequence of the material properties and the solution of the PD theory. The material properties are listed in Table 1 which are similar to those used in the experiments of Gondret et al. [27]. The boundary conditions of the plate also affect the values of $T_{c}$ and $r_{c}$. Therefore, we test a plate with a free and a fixed boundary condition at the bottom. For both cases the lateral boundaries are fixed and the upper boundary is free. The impact velocity in 235 the absence of any fluid is then $V_{i m p}=21 \mathrm{~cm} / \mathrm{s}$. For the free boundary case the material responds to the impactor resulting in $r_{c}=0.44$ which is far from 0.97. However, for a fixed bottom boundary, we calculated $r_{c}=0.97$ in accordance with the value from Ardekani and Rangel [26] and the contact duration $T_{c}$ is 7 $\mu \mathrm{s}$.

Because there is only one rigid particle in the field and no collision between rigid particles will happen, we do not need to consider $\delta t_{c}^{d e m}$. The influence of the number of sub-iterations of PD $N$ on the simulation is investigated by taking $N=(10,15,20,25)$ and $\delta t^{p d}=0.3 \delta t_{c}^{p d}=2.6 \times 10^{-9}$ s, leading to a time step $\delta t=N \delta t^{p d}$. Our validation also shows the difference between rebounds 245 happening on a rigid plate (DEM) and a deformable plate (PD), where $r_{c}$ and $T_{c}$ of DEM are the same as those of PD.

Figure 4 shows that the DEM results agree well with the data reported by Ardekani and Rangel [26, where the collision happens on a rigid plate. For the deformable plate resolved with PD, the velocity after the first rebound is slightly 
Table 1: The physical properties of material and impactor.

\begin{tabular}{llll}
\hline Material Properties & & & \\
\hline Length & $L$ & $\mathrm{~m}$ & 0.03 \\
Width & $W$ & $\mathrm{~m}$ & 0.006 \\
Thickness & $h$ & $\mathrm{~m}$ & $6 \times 10^{-5}$ \\
Young's module & $E$ & $\mathrm{Gpa}$ & 55 \\
Poisson's ratio & $\nu$ & 1 & 0.3333 \\
Density & $\rho_{m}$ & $\mathrm{~kg} / \mathrm{m}^{3}$ & 2500 \\
\hline Impactor & & & \\
\hline Diameter & $D$ & $\mathrm{~m}$ & 0.003 \\
Density & $\rho_{p}$ & $\mathrm{~kg} / \mathrm{m}^{3}$ & 7800 \\
\hline
\end{tabular}

250 smaller when $N=25$, while it is larger when $N=(10,15,20)$. Particularly, the difference between the cases with $N=(10,15,20)$ at the first rebound is quite small. Therefore we opt for the smaller $N=10$ and $\delta t^{p d}=0.3 \delta t_{c}^{p d}$ to ensure the accuracy of the following simulations. The vortex distribution and the velocity field of the material are displayed in Figure 5. It shows the material response to the particle impact in a viscous fluid. It demonstrates the importance of employing proper material models when investigating particlewall collision dynamics in a viscous fluid.

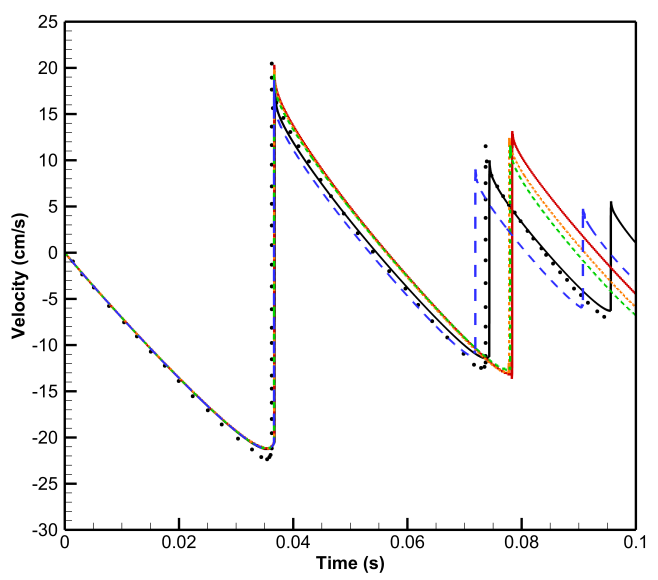

Figure 4: Time history of the vertical velocity of the particle moving in fluid. The results from Ardekani and Rangel [26]: •-; DEM: - $\longrightarrow$ PD, $N=10$ : - ; PD, $N=15$ : ........; PD, $N=20: \cdots ;$ PD $, N=25: \cdots$ 

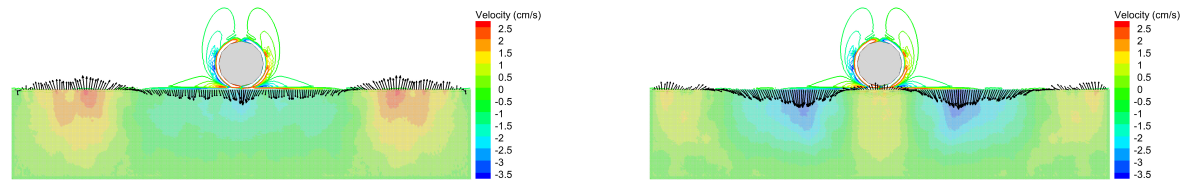

Figure 5: The vortex distribution, the vertical velocity field of the material plate, and the velocity vectors of the IB points on the surface of the material plate during the first rebound.

\subsection{Particle-wall impact in the absence of a fluid}

In this section, a rigid particle colliding into the middle of a brittle wall at a right angle is simulated to validate the PD solver for material damage prediction. Here, the implemented PD solver will naturally capture the material crack. The diameters $D$ of the rigid particles are $1 \mathrm{~mm}$ and $12.5 \mathrm{~mm}$, we compare our simulation results with the experimental data of the impact of a small steel sphere against the glass surface 28. The material properties are listed in Table 1 and $D=50 \Delta$. The target's length and width are set to $L=10 D$ and $W=2 D$ respectively and the thickness is $h=0.02 D$. As the experimental specimen of Ref. 28 is relatively large and fixed on a plane, we fix the bottom and the lateral boundaries of the target material. Considering the difference in dimensions, material size and impactor's shape, the fracture energy $G_{c}$ is chosen 270 to be $342 \mathrm{~J} / \mathrm{m}^{2}$ and $96 \mathrm{~J} / \mathrm{m}^{2}$ for the particles with diameters $1 \mathrm{~mm}$ and 12.5 $\mathrm{mm}$, respectively.

As shown in Fig. 6, our simulation results agree well with the experimental data reported by Knight et al. 28. For rigid particles with different sizes, we found that the restitution coefficient $r_{c}$ becomes smaller by increasing the impact velocity. Moreover, the material damages caused by both the rigid impactors with different impact velocities are displayed in Fig. 77. It is in accordance with the experiment of Knight et al. 28 that a particle with larger size and impact velocity tends to make a much more significant damage. Fracture appears quickly when the rigid particle comes into contact with the surface in a relatively large impact velocity. The crack patterns caused by the normal dynamic loading of the rigid particles are displayed in Fig. 8 and Fig. 9 , respectively. 


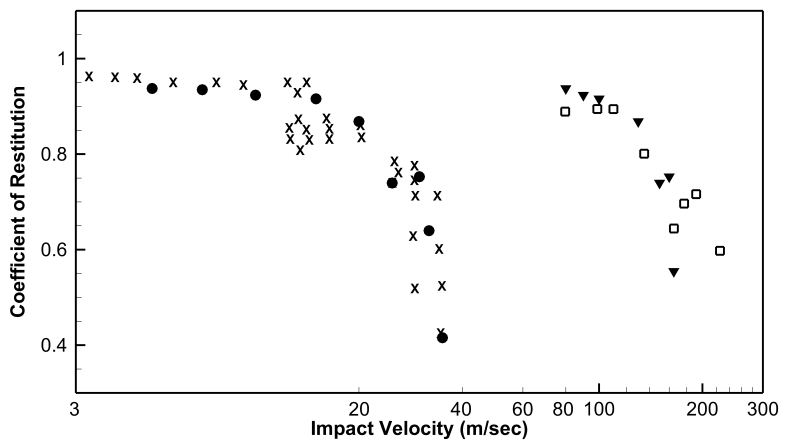

Figure 6: Variations of the restitution coefficient $r_{c}$ with impact velocity $V_{\text {imp }}$ for rigid particles of size $1 \mathrm{~mm}$ and $12.5 \mathrm{~mm}$. Knight et al. 28] experimental results are presented alongside the simulations results. Ref. 28]: $1 \mathrm{~mm}\left(\right.$ (), $12.5 \mathrm{~mm}\left({ }^{(}\right)$; the present results: $1 \mathrm{~mm}(\mathbf{\nabla}), 12.5 \mathrm{~mm}$ (•)

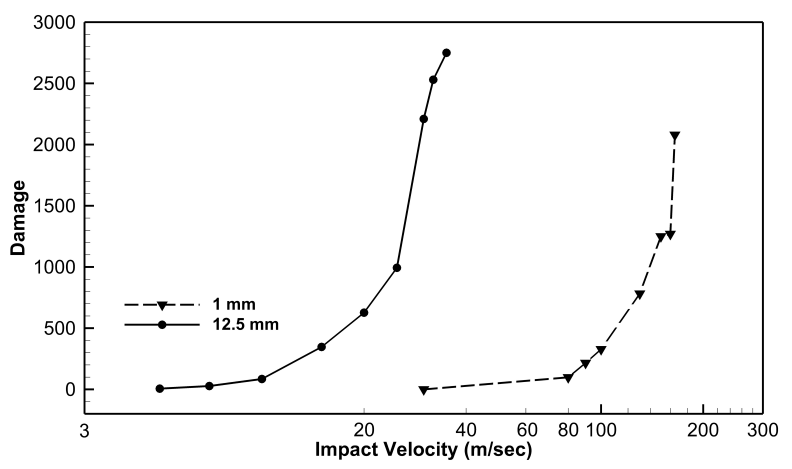

Figure 7: Variations of the total damage $D_{t o t}$ as a function of the impact velocity $V_{\text {imp }}$.

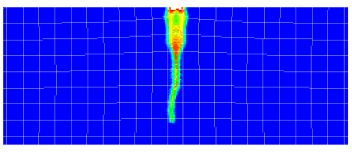

(a) $V_{i m p}=15 \mathrm{~m} / \mathrm{s}$

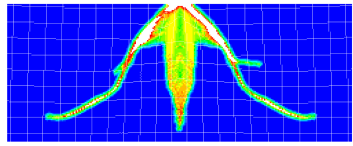

(b) $V_{i m p}=30 \mathrm{~m} / \mathrm{s}$

Figure 8: The crack patterns caused by a rigid particle of size $12.5 \mathrm{~mm}$. 


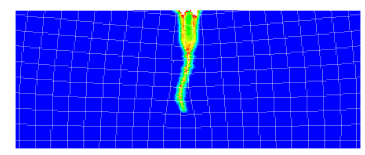

(a) $V_{i m p}=100 \mathrm{~m} / \mathrm{s}$

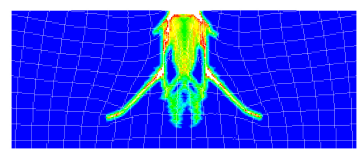

(b) $V_{i m p}=150 \mathrm{~m} / \mathrm{s}$

Figure 9: The crack patterns caused by a rigid particle of size $1 \mathrm{~mm}$.

The results presented in Fig. 6 and Fig. 7 demonstrate that at relatively low impact velocities, the restitution coefficient is high and the damage is negligible. In practice, there are many situations that material damage and erosion 285 become visible gradually during the continuous impacts of many particles under a relatively low $V_{i m p}$. The erosion due to the impacts occurs over months and the damage accumulates with the number of impacts. To study the erosion phenomena, we increase the number of particle impacts against a wall in a short time duration. A particle of size $1 \mathrm{~mm}$ and $V_{i m p}=30 \mathrm{~m} / \mathrm{s}$ is forced to hit the surface continuously. Once the particle bounces away from the surface, it will be accelerated to $V_{i m p}=30 \mathrm{~m} / \mathrm{s}$ in an opposite direction to hit the surface again. The evolution of the material damage is displayed in Fig. 10, It shows that in the early stage these impacts produce little damage but after a critical number of collisions $(\approx 12)$ the material fails and the damage grows at an 295 exponential-like rate until approximately 21 collisions where the rate of damage becomes linear. During this process a crater on the material surface forms and gradually grows with the fractures inside the material, see in Fig. 11.

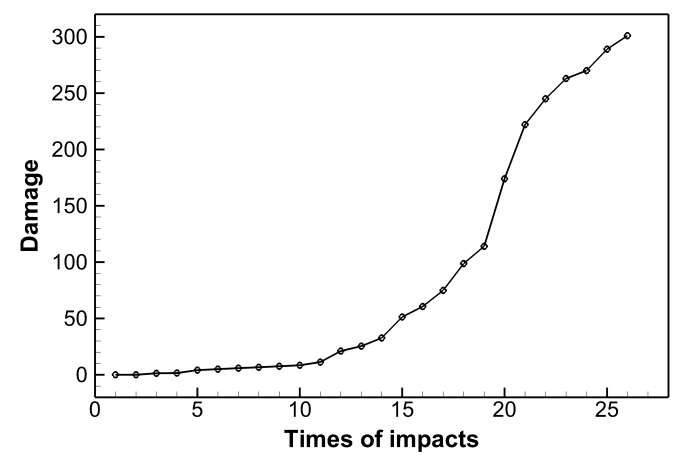

Figure 10: The total damage of material $D_{\text {tot }}$ produced by the continuous normal impacts from a rigid particle of size $1 \mathrm{~mm}$ with $V_{i m p}=30 \mathrm{~m} / \mathrm{s}$. 


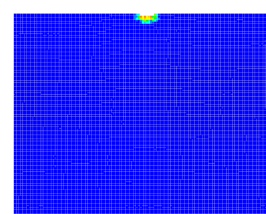

(a) $M=12$

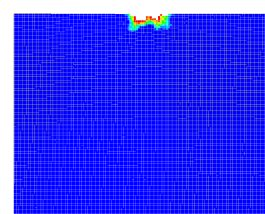

(b) $M=17$

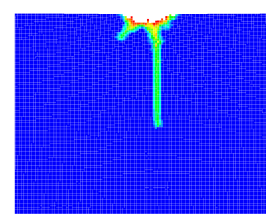

(c) $M=21$

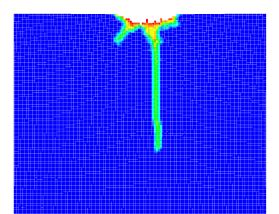

(d) $M=25$

Figure 11: The crater formed after $M$ normal impacts from a rigid particle of size $1 \mathrm{~mm}$ with $V_{i m p}=30 \mathrm{~m} / \mathrm{s}$.

\section{Erosion caused by particle-wall collision in a viscous fluid}

As indicated by Finnie [3] the erosion caused by solid particles in a fluid depends on both the mechanism of material damage and the fluid flow. The surrounding fluid flow significantly affects the motion of the impactor. The drag force, added mass and the Basset history effects should be considered particularly for particles' motion in a fluid with higher viscosities and densities. Our current method is capable of direct evaluation of the material damage and hydrodynamic effects simultaneously without any empirical models. In this section, several simulations will be conducted to demonstrate the capability of the new method for resolving the interactions between the material damage and fluid flow.

In this section, we consider the erosion caused by single/multiple particlewall collisions using the set-up presented in Figure 3 . The amount of material erosion is represented by the total damage to the materials. The properties of the material are summarized in Table 1. The particle is released with a given impact angle $\alpha$ and an initial velocity $V_{i}$ at a certain distance away from the predicted hit point. A liquid-like and a gas-like fluid are considered and their 315 densities $\rho$ and kinematic viscosities $\nu$ are respectively set to $0.935 \mathrm{~g} / \mathrm{cm}^{3}$ and $0.1 \mathrm{~cm}^{2} / \mathrm{s}, 0.001 \mathrm{~g} / \mathrm{cm}^{3}$ and $0.15 \mathrm{~cm}^{2} / \mathrm{s}$.

\subsection{Single-particle impact}

A rigid particle with a diameter $D=12.5 \mathrm{~mm}, V_{i}=15 \mathrm{~m} / \mathrm{s}$ and $V_{i}=30$ $\mathrm{m} / \mathrm{s}$ is released normally $\left(\alpha=90^{\circ}\right)$ at a distance $1.5 D$ away from the surface . The size of computational domain in Fig. 3 is $(10 D, 10 D)$. The length and width of the target wall are set to $10 D$ and $2 D$ respectively.

The velocity and the direction of particles colliding with a surface are largely determined by the flow conditions. The vertical velocities of particles released in both media are plotted in Fig. 12a. For the liquid, the initial velocities of 15 $\mathrm{m} / \mathrm{s}$ and $30 \mathrm{~m} / \mathrm{s}$ drop rapidly when the particle is released. Then, the particle velocity decreases more slowly until reaching the impact velocities $V_{i m p}$ of 10.6 $\mathrm{m} / \mathrm{s}$ and $21.2 \mathrm{~m} / \mathrm{s}$, respectively. Their corresponding restitution coefficients are 0.93 and 0.80 . For the gas medium, the particle velocities remain nearly the same, $V_{i m p} \approx V_{i}$. The restitution coefficients $r_{c}$ are about 0.79 and 0.69 , which 
is lower than the values in the liquid phase. This appears to be related to the higher damage to the surface during the collision in the gas-like fluid. Fig. 12b shows the material damage growth during loading and unloading of the impact.

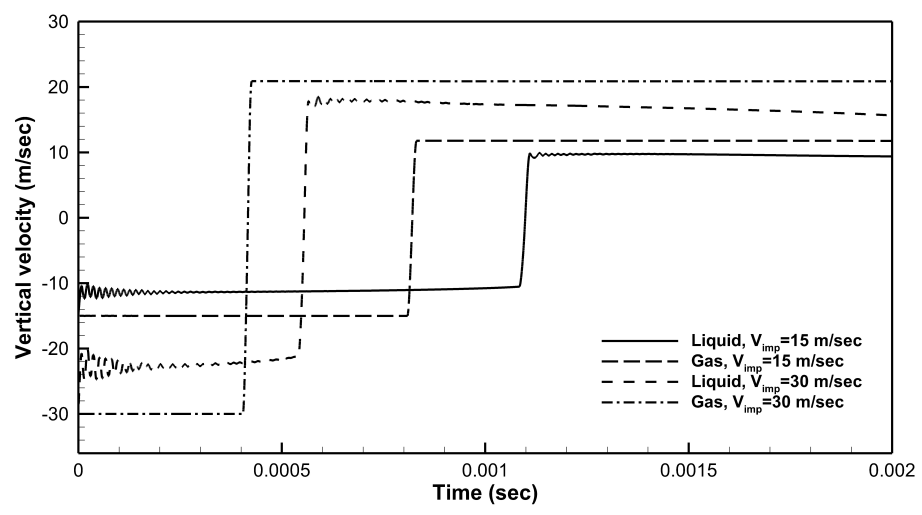

(a)

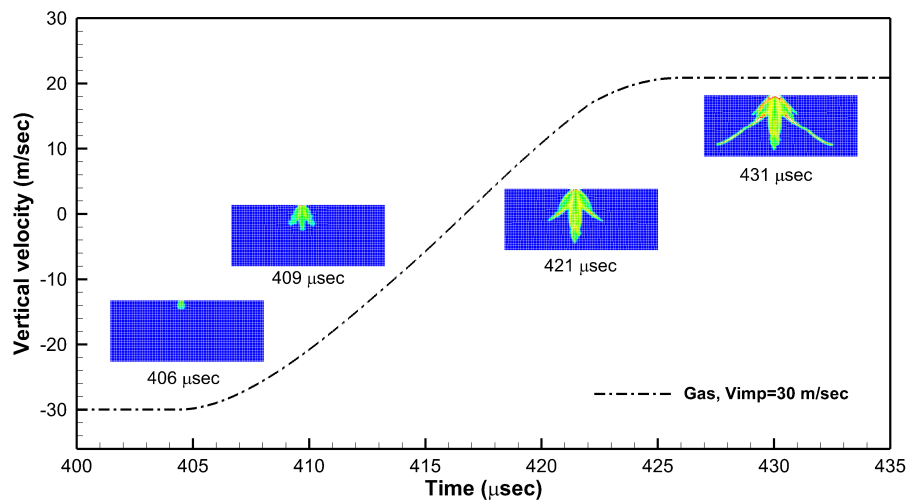

(b)

Figure 12: (a) the vertical velocities of the rigid particle of size $12.5 \mathrm{~mm}$ in the liquid and the gas media; (b) the material damage development during loading (impact) and unloading (rebound).

Fig. 13 and Fig. 14 display the vortex distributions of the flow field at the moments when the particle moves to a similar height, along with the material damage produced during the impact. For the impact shown in Fig. 13a, after the particle bounces away from the surface, three pairs of vortices appear, which are marked with $V 1, V 2$ and $V 3$. While, $V 1$ move upward with the bouncing particle and its right part begins to shed, $V 3$ move away from the impact point 340 along the material surface, the weakest pair $V 2$ appear in the area between particle and wall and move to the left. Fig. 13b shows the collision of a particle with a higher release velocity of $V_{i}=30 \mathrm{~m} / \mathrm{s}$ which as expected, causes a larger 
damage and more asymmetric vortices appear in the system after the collision. Similar behaviour is observed in Figure 14 for the collision in a liquid-like fluid.

345 Generally, since the impact velocity is smaller for the same release velocity in the liquid-like fluid, less damage is observed. However, in all the cases the particle is deflected to the left with a vortex being shed to the opposite direction, e.g. see Fig. $14 \mathrm{~b}$.

It is also worth mentioning that the deflection is triggered by the details of the damage to the surface and is expected due to the physically unstable nature of the rebound where any microscopic defect on the surface or material inhomogeneity will result in an asymmetrical damage and impact forces which consequently deflects the particle's trajectory. However, in the simulations, the instabilities are generated by the details of the numerical schemes such as rounding errors and convergence criteria. Therefore, the deflection appears to be deterministic and systematically to the left in all the cases.
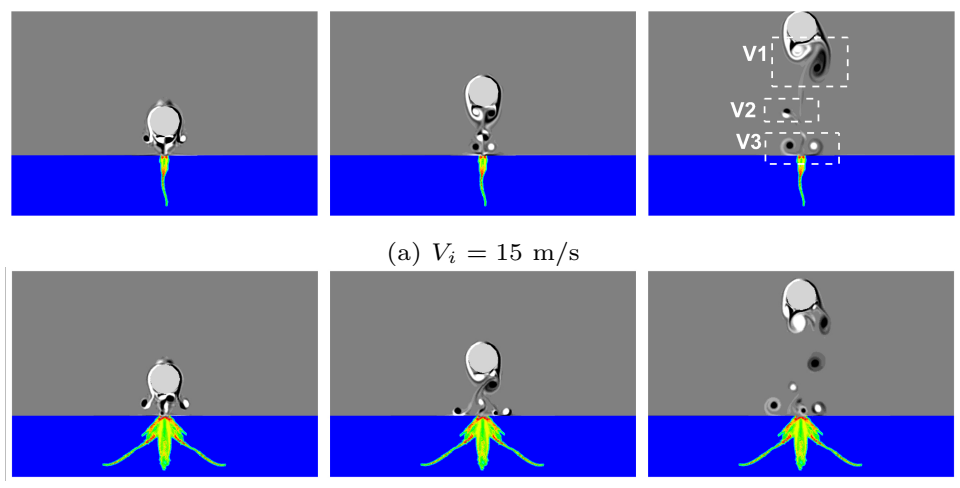

(a) $V_{i}=15 \mathrm{~m} / \mathrm{s}$
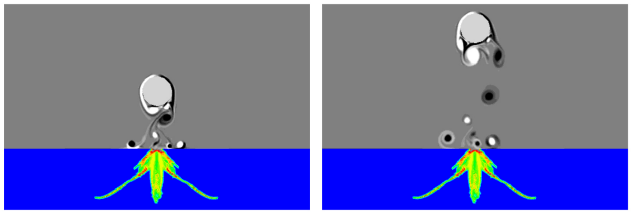

(b) $V_{i}=30 \mathrm{~m} / \mathrm{s}$

Figure 13: The vortex pairs induced by a single particle-wall collision with an impactor of size $12.5 \mathrm{~mm}$ in a gas-like fluid. 

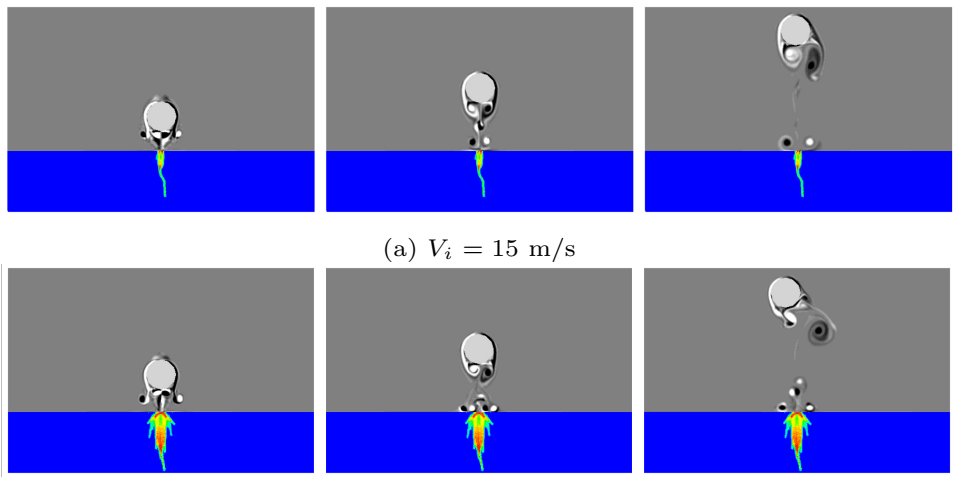

(b) $V_{i}=30 \mathrm{~m} / \mathrm{s}$

Figure 14: The vortex pairs induced by a single particle-wall collision with an impactor of size $12.5 \mathrm{~mm}$ in a liquid-like fluid.

\subsection{Erosion produced by multiple-particle impacts}

So far only the normal impact of a single particle is considered. The vast majority of impacts in engineering systems of interest, however, take place at an angle to the surface. Therefore, multi-particle collisions with a surface at varying impact angles are investigated in this section. The computational domain used in the previous sections is enlarged to $(20 D, 16 D)$ and accordingly, the size of the target wall is set to $(20 D, 2 D)$ to avoid any contact between rigid particles which could deflect them from their principal moving path. The distance between rigid particles is $1.4 D$. The influence of the number of impactors $M$ and angles $\alpha$ on the erosion of a brittle material is studied. The predicted hit point is at $(10 D, 0)$. Six rigid particles of size $D=12.5 \mathrm{~mm}$ are released in the gas with $V_{i}=11.3 \mathrm{~m} / \mathrm{s}$ and impact angles $\alpha=\left(30^{\circ}, 45^{\circ}, 60^{\circ}\right)$.

The material damages caused by several particle collisions are recorded in 370 Figure 15, which shows that the material damages increase with the number of collisions, $M$. Moreover, a larger impact angle $\alpha$ produces a more severe damage which is in agreement with Zheng et al. 10] and Bitter 29] who showed that brittle material usually suffers a peak erosion during normal impacts. The induced vortices and the caused material damages by six rigid impactors are presented in Fig. 16. Similar to the single particle impacts (e.g. see Fig. 11), a crater emerges on the surface as a result of the erosion and expands gradually while the fractures penetrate deeper inside the material. 


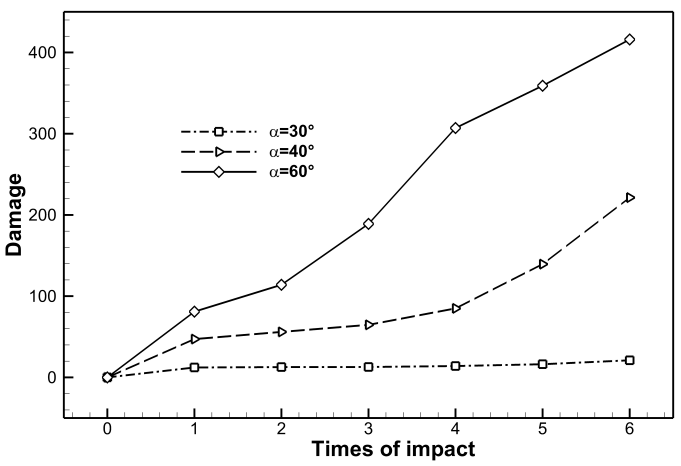

Figure 15: The total damage of material $D_{\text {tot }}$ after $M$ normal impacts. 

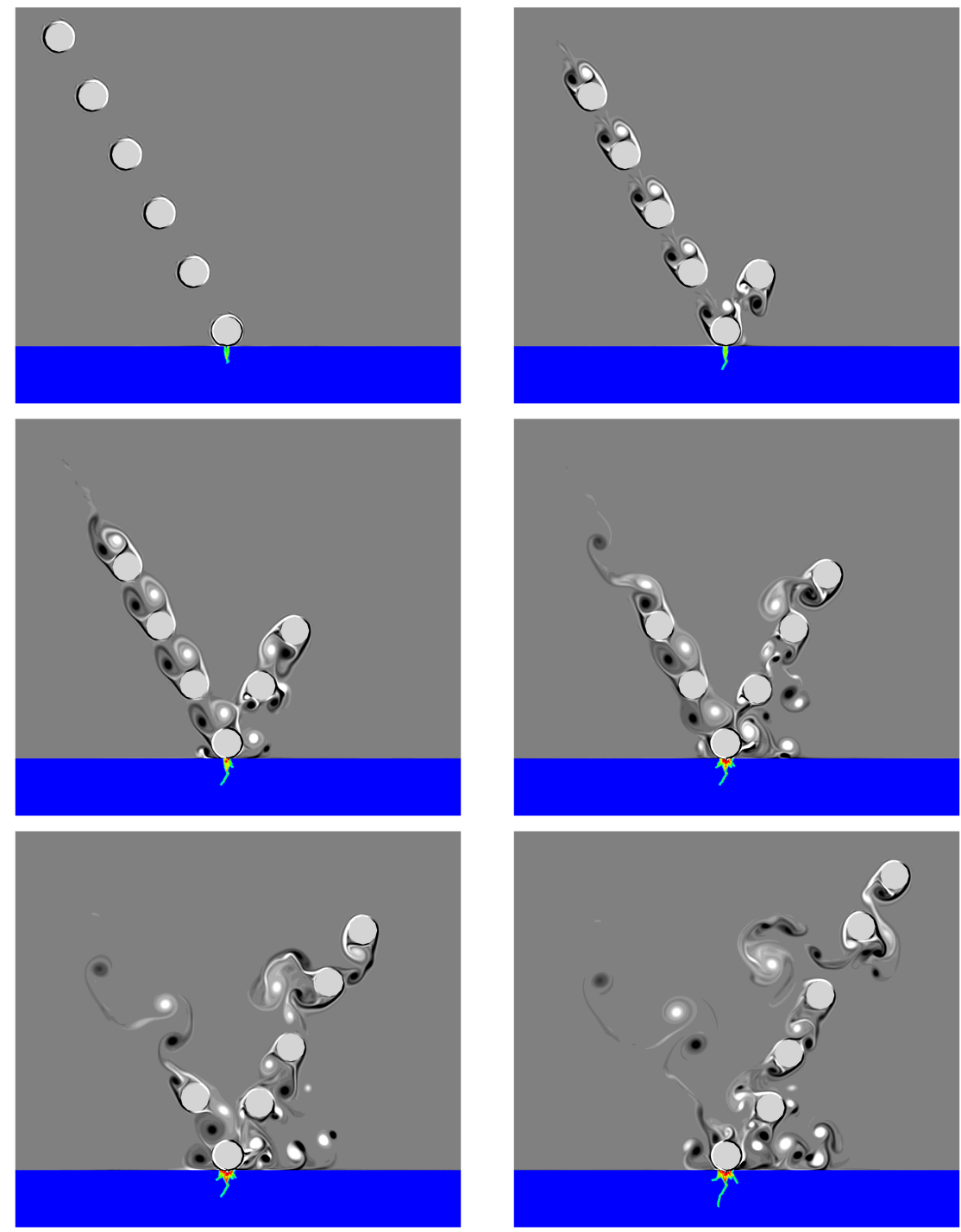

Figure 16: The vortices and the material damage under number of impacts (1-6) from impactors of size $12.5 \mathrm{~mm}$ with $\alpha=60^{\circ}$ and $V_{i}=11.3 \mathrm{~m} / \mathrm{s}$.

\section{Conclusions}

In this paper, a novel algorithm is presented by combining the peridynamics theory and DEM-IB-CLBM approach to fully resolve the material erosion caused by the collision of solid particles. The fully resolved fluid-particle coupling (DEM-IB-CLBM approach) provides accurate trajectories of the particles and their rotational and translational kinetic energies just before the impact. 
Meanwhile, the peridynamics theory directly estimates the material damage. The coupled method is capable of providing invaluable information about the erosion mechanisms due to the particle impacts which is not available through any other simulation technique. Several benchmark problems are considered to demonstrate accuracy of the method including single particle impact simulations in liquids and gasses. Furthermore, it is demonstrated that the current 390 approach is capable of predicting the influence of the impact angle on the erosion rate and can effectively capture the growth of damage due to multiple impacts which results in the formation of a crater on the surface.

\section{Acknowledgment}

This work is supported by the National Natural Science Foundation of China 395 (Grant No. 11502210, 51709229, 51879220, 51479170 and 61803306); the National Key Research and Development Program of China (Grant No. 2016YFC0301300);

and the Natural Science Basic Research Plan in Shaanxi Province of China (Grant No. 2018JQ5092).

\section{References}

[1] I. Hutchings, A model for the erosion of metals by spherical particles at normal incidence, Wear 70 (1981) 269-81.

[2] S. Walley, J. Field, The contribution of the Cavendish Laboratory to the understanding of solid particle erosion mechanisms, Wear 258 (2005) 55266 .

[3] I. Finnie, Erosion of surfaces by solid particles, Wear 3 (1960) 87-103.

[4] R. D. Aponte, L. A. Teran, J. A. Ladino, F. Larrahondo, J. J. Coronado, S. A. Rodríguez, Computational study of the particle size effect on a jet erosion wear device, Wear 374-375 (2017) 97-103.

[5] R. E. Vieira, A. Mansouri, B. S. McLaury, S. A. Shirazi, Experimental and computational study of erosion in elbows due to sand particles in air flow, Powder Technology 288 (2016) 339-53.

[6] H. Liu, Z. Zhou, M. Liu, A probability model of predicting the sand erosion profile in elbows for gas flow, Wear 342-343 (2015) 377-90.

[7] B. Lee, J. Tu, C. Fletcher, On numerical modeling of particle-wall impaction in relation to erosion prediction: Eulerian versus Lagrangian method, Wear 252 (2002) 179-88.

[8] M. Parsi, K. Najmi, F. Najafifard, S. Hassani, B. S. McLaury, S. A. Shirazi, A comprehensive review of solid particle erosion modeling for oil and gas wells and pipelines applications, Journal of Natural Gas Science and Engineering 21 (2014) 850-73. 
[9] Y. Ben-Ami, A. Uzi, A. Levy, Modelling the particles impingement angle to produce maximum erosion, Powder Technology 301 (2016) 1032-43.

[10] C. Zheng, Y. Liu, C. Chen, J. Qin, R. Ji, B. Cai, Numerical study of impact erosion of multiple solid particle, Applied Surface Science 423 (2017) 17684.

[11] Y. Zhang, Y. Zhang, G. Pan, S. Haeri, Numerical study of the particle sedimentation in a viscous fluid using a coupled DEM-IB-CLBM approach, Journal of Computational Physics 368 (2018) 1-20.

[12] S. Silling, Reformulation of elasticity theory for discontinuities and longrange forces, Journal of the Mechanics and Physics of Solids 48 (2000) $175-209$.

[13] S. A. Silling, E. Askari, Peridynamic Modeling of Impact Damage, in: Problems Involving Thermal Hydraulics, Liquid Sloshing, and Extreme Loads on Structures, volume 2004, ASME, 2004, pp. 197-205.

[14] S. A. Silling, E. Askari, A meshfree method based on the peridynamic model of solid mechanics, Computers and Structures 83 (2005) 1526-35.

[15] E. Oterkus, I. Guven, E. Madenci, Impact damage assessment by using peridynamic theory, Central European Journal of Engineering 2 (2012) 523-31.

[16] M. R. Tupek, Extension of the peridynamic theory of solids for the simulation of materials under extreme loadings, Ph.D. thesis, Massachusetts Institute of Technology, 2014.

[17] E. Madenci, S. Oterkus, Ordinary state-based peridynamics for plastic deformation according to von Mises yield criteria with isotropic hardening, Journal of the Mechanics and Physics of Solids 86 (2016) 192-219.

[18] E. Madenci, E. Oterkus, Peridynamic Theory and Its Applications, volume 9781461484, Springer New York, New York, NY, 2014.

[19] K. N. Premnath, S. Banerjee, Incorporating forcing terms in cascaded lattice Boltzmann approach by method of central moments, Physical Review E - Statistical, Nonlinear, and Soft Matter Physics 80 (2009).

[20] S. Haeri, J. S. Shrimpton, On the application of immersed boundary, fictitious domain and body-conformal mesh methods to many particle multiphase flows, International Journal of Multiphase Flow 40 (2012) 38-55.

[21] S. Haeri, J. S. Shrimpton, A new implicit fictitious domain method for the simulation of flow in complex geometries with heat transfer, Journal of Computational Physics 237 (2013) 21-45. 
[22] S. Haeri, J. S. Shrimpton, Fully resolved simulation of particle deposition and heat transfer in a differentially heated cavity, International Journal of Heat and Fluid Flow 50 (2014) 1-15.

460

[29] J. Bitter, A study of erosion phenomena, Part II, Wear 6 (1963) 169-90. 